\title{
Computer Assisted Planning for Curved Laser Interstitial Thermal Therapy
}

\author{
M. Pinzi, V. N. Vakharia, B. Y. Hwang, W. S. Anderson, J. S. Duncan, F. Rodriguez y Baena
}

\begin{abstract}
Laser interstitial thermal therapy (LiTT) is a minimally invasive alternative to conventional open surgery for drugresistant focal mesial temporal lobe epilepsy (MTLE). Recent studies suggest that higher seizure freedom rates are correlated with maximal ablation of the mesial hippocampal head, whilst sparing of the parahippocampal gyrus (PHG) may reduce neuropsychological sequelae. Current commercially available laser catheters are inserted following manually planned straight-line trajectories, which cannot conform to curved brain structures, such as the hippocampus, without causing collateral damage or requiring multiple insertions. Objectives: The clinical feasibility and potential of curved LiTT trajectories through steerable needles has yet to be investigated. This is the focus of our work. Methods: We propose a GPU-accelerated computer-assisted planning (CAP) algorithm for steerable needle insertions that generates optimized curved 3D trajectories with maximal ablation of the amygdalohippocampal complex and minimal collateral damage to nearby structures, while accounting for a variable ablation diameter $(5-15 \mathrm{~mm})$. Results: Simulated trajectories and ablations were performed on 5 patients with mesial temporal sclerosis (MTS), which were identified from a prospectively managed database. The algorithm generated obstacle-free paths with significantly greater target area ablation coverage and lower PHG ablation variance compared to straight line trajectories. Conclusions: The presented CAP algorithm returns increased ablation of the amygdalohippocampal complex, with lower patient risk scores compared to straight-line trajectories. Significance: This is the first clinical application of preoperative planning for steerable needle based LiTT. This study suggests that steerable needles have the potential to improve LiTT procedure efficacy whilst improving the safety and should thus be investigated further.
\end{abstract}

Index Terms-computer assisted planning, steerable needles, neurosurgery, LiTT, epilepsy surgery

\section{INTRODUCTION}

Several surgical disciplines have seen significant advances in the last decade, with the introduction of novel robotic and endoscopic tools that have aided more extensive resections through minimally invasive corridors [1], [2]. Amongst these, steerable needle technologies have been tested for different neurosurgical applications. Stereotactic needle-based brain biopsies [3], deep brain stimulation [4], [5], [6], stereoelectroencephalography, stereotactic needle-based aspiration [7], stereotactic brachytherapy [8] and intracerebral drug delivery [9], [10] could all potentially benefit from steerable needle technologies. Another needle steering application, yet to be investigated, is laser interstitial thermal therapy (LiTT). LiTT provides a minimally invasive alternative to conventional open surgery [11] for drug-resistant mesial temporal

This work was supported by the European Union's Horizon 2020 Research and Innovation Action under grant agreement No 688279 (EDEN2020). lobe epilepsy (MTLE), which is the most common drugrefractory focal epilepsy [12]. Selective amygdalohippocampal complex (AHC) ablation with LiTT is currently performed by means of a straight catheter featuring a laser tip which is inserted through the brain along the longitudinal axis of the hippocampus to the anterior border of the amygdala. The extend of the ablation can be modulated through MRthermography to generate an ablation diameter between 5 to $15 \mathrm{~mm}$. Recent studies demonstrated that post-ablation seizure freedom (defined as "Engel I" outcome) are correlated with maximal ablation of the mesial hippocampal head, amygdala and the entorhinal cortex (EnCx), while sparing of the parahippocampal gyrus (PHG) and collateral structures may reduce the probability of neuropsychological complications [13], [14]. Additionally, a multi-center validation study by Galovic et al. [15] supports the importance of resecting the temporal portion of the piriform cortex $(\mathrm{PiCx})$ during temporal lobectomy, resulting in increased odds of achieving seizure freedom by a factor of 16. The most common LiTT-related neurological complications are visual field deficits, ranging from 5 to $29 \%$ of total cases [16], [17]. Visual deficits arise from heat transfer to the optic radiation or the lateral geniculate nucleus (LGN) during the ablation process.

Automated computer assisted planning (CAP) for straight tools has been proposed by Vakharia et.al [18], [19] to generate optimized paths that maximize AHC ablation, spare the PHG and maintain a safety distance from the brainstem, LGN, sulci and vasculature. The machine learning-based method was validated in a multi-centre retrospective study [20] in which three automated straight trajectories and corresponding outcomes, featuring different entry point and target constraints, were compared to manually planned and implemented paths in 95 MTLE patients. The results showed a significant improvement in safety parameters and amygdalohippocampal complex ablation volumes and blinded external expert reviewers preferred the CAP trajectories over the manually planned trajectories.

However, the hippocampus features a distinctive curved shape that is generally very challenging to ablate in one single trajectory without damaging nearby structures, avoiding vasculature, transgressing the ventricular ependyma, as well as not being feasible in patients with abnormal anatomy. A trade-off between total target ablation coverage and minimum collateral damage is difficult to achieve even for automated methods based on straight tools. In some circumstances, more than one trajectory is necessary to achieve a successful ablation [21], thus increasing operative time and surgical risk. Additionally, a suboptimal ablation, due to the limited flexibility of currently available laser tools, resulted in $46 \%$ of patients requiring 
follow-on surgeries to achieve seizure freedom in some series [22].

A curved trajectory through a steerable needle could provide a better trade-off between coverage of the target area and damage reduction to collateral structures, while facilitating the inclusion of $\mathrm{EnCx}$ and $\mathrm{PiCx}$, as recently reported in terms of Engel I outcomes for correlated structures [16], [23]. D.Comber et al [24] proposed a patient-specific design of concentric tube needles for a transforamenal approach to access the hippocampus. In this study, for each of the 20 selected hippocampi, a concentric tube robot was designed and optimized to traverse a trajectory from the foramen ovale to and through the hippocampus, from head to tail. Patient-specific needles were rapidly fabricated featuring a precurved of up to $32.4 \mathrm{~mm}^{-1}$. However, the insertion path was selected to simply match the hippocampus centerline and ablate the AHC. Consequently, to date, there are no studies proposing the computation of curvature-bounded optimized paths for steerable needles in the context of LiTT, taking into account ablation parameters and the optimization of chance with respect to seizure-free outcomes. Here, we propose a computer assisted planning (CAP) algorithm able to maximize $\mathrm{AHC}, \mathrm{EnCx}$ and PiCx ablation whilst minimizing damage to the PHG and surrounding structures. Surgeons will be able to fine-tune LiTT related parameters during the preoperative phase and interactively assess the generated path looking at the corresponding expected ablation of brain structures, both in the standard surgical $2 \mathrm{D}$ view and in a 3D rendering. In this work, a three-dimensional path planner for systems with nonholonomic constraints in complex environments is developed. A Bevel tip needle is considered to be a nonholonomic system, that is to say, one with nonintegrable velocity constraints [25]. Our own design is that of a programmable bevel tip needle (PBN) [26], which is inspired by the multi-segment ovipositor of certain parasitic wasps. The needle consists of four interlocked segments forming a thin and flexible shaft ending in a bevel tip. A programmable offset between the segments, which are made of flexible plastic and thus fully Magnetic Resonance Imaging (MRI) compatible, is used to steer the tip dynamically within a softer medium. PBNs are able to steer along three-dimensional paths without duty cycle spinning along the insertion axis (as in [27]), and thus offer an ideal solution for the path planning technique described in this work. This needle design has been the focus of EDEN2020, a Horizon 2020 funded project that demonstrated the planning and control of a $3.5 \mathrm{~mm}$ PBN prototype during in-vivo and ex-vivo animal trials [28], [29].

With this study we aim to show the potential of steerable needles, such as PBN involved in our EDEN2020 trials, to improve the efficacy of LiTT procedures whilst improving safety. This is the first clinical application of preoperative planning for steerable-needle-based LiTT. Among its novel features we highlight:(1) A GPU accelerated algorithm for patient-specific optimisation of curved LiTT ablation volume; (2) Ablation of the piriform temporal portion $(\mathrm{PiCx})$, which is included for the first time in a LiTT CAP assessment; (3) Optimisation of the laser ablation diameter for each ablation step to achieve more precise targeting of the lesion; (4) The introduction of a new combined cost function for curved LiTT to rank the generated paths with respect to both patient risk and path smoothness.

Finally, we assess generated curved CAP LiTT trajectories on five patients with hippocampal sclerosis (HS) and compare the results of target structure ablation (Amygdala, Hippocampus, $\mathrm{EnCx}$ and $\mathrm{PiCx}$ ), collateral damage (temporal white matter, PHG and fusiform gyrus) and associated trajectory risk metrics with respect to a previously published straight CAP LiTT method [20], [30].

\section{Methodology}

\section{A. Curved Computer Assisted Planning}

In this study we introduce a novel curved LiTT CAP which optimizes a path for mesial temporal lobe epilepsy treatment. The principal mechanism behind LiTT is to selectively ablate tissue of interest by heat produced from an optical fiber. The optical fiber is inserted into the mesial temporal lobe through a small hole made in the skull and its location is confirmed under MRI guidance. The tip of the laser fiber produces heat, resulting in an approximately spherical ablation of the surrounding target tissue area. The fiber is then pulled back in small interment steps and the procedure is repeated to cover the complete target volume. Our method aims to minimize path length, overall curvature, ablation of PHG and surrounding critical structures such as the LGN, fusiform gyrus $(\mathrm{FuG})$ and temporal white matter (WM). On the other hand, the algorithm maximizes the ablation of the hippocampal head and body, amygdala, $\mathrm{EnCx}$ and the $\mathrm{PiCx}$, which are structures correlated with the Engel 1 outcome in MTLE patients [16], [23]. Trajectories that do not meet the constraint of a 120 mm maximum length and $\rho=\frac{1}{35} \mathrm{~mm}^{-1}$ maximum achievable needle curvature are rejected. The latter was chosen on the basis of a previous study [31], which estimated the average curvature of the AHC complex for steerable needle designs to have a radius of $49.015 \pm 14.79 \mathrm{~mm}$, from which we derived a reasonable minimum radius of $35 \mathrm{~mm}$. Furthermore, simulation and experimental results on the EDEN2020 PBN [32], [33] show that similar curvature can be reached by increasing the needle stiffness, reducing its size and tuning the tip offset [33]. Paths colliding with obstacles such as the atrium of the lateral ventricles, vasculature and sulci, or less than $7.5 \mathrm{~mm}$ away from the brainstem and LGN, are also rejected, as in [19], [20], to prevent excess heat transmission.

The distance from critical structures such as the vasculature and sulci is optimized by means of a risk score, first introduced in [18], [19]. This metric, normalized to be within the $[0,1]$ range, measures the overall path risk as the cumulative distance from critical structures along the entire intracerebral trajectory. The minimum distance here is set to be $3 \mathrm{~mm}$, as in Li et al. [19], so that a path having a risk score equal to zero is constantly at more than $10 \mathrm{~mm}$ distance from obstacles, while a risk of 1 corresponds to a path being continuously at $3 \mathrm{~mm}$ from obstacles.

The proposed CAP consists of a combination of two different planners. The first one addresses the optimization of the portion of the path in correspondence of the target ablation 
area, which we will refer to from now as the "ablation path". It aims to calculate a trajectory which optimizes the overall ablation of the targeted area, while reducing collateral tissue damage. The second is based on the previously presented adaptive fractal tree [34] and adaptive hèrmite fractal tree (AHFT) [35] path planner techniques and aims to find a curvature-bounded obstacles-free path to connect the ablation path to a suitable entry point on the patient skull; we will refer to this portion of the path as "AFT-connection".

1) Ablation Path: The first step is novel method that involves the generation of a so-called "ablation field map" (Figure 1). A GPU accelerated algorithm evaluates each voxel of the obstacle map in parallel. On each thread, given an homogeneous ablation diameter $D_{a}$, a sphere centered on a specific voxel is considered. The search space within the sphere is evaluated; the number of voxels belonging to obstacle regions $N_{d}$ are counted as unwanted tissue damage; the voxels belonging to the ablation target area $N_{a}$ are counted as successful tissue ablation; while the voxels not belonging to the previous categories are counted as additional tissue damage $N_{r}$. A normalized score $S_{v}$ in the range [0,1], computed as the weighted positive contribution of the percentage of damage and additional tissue ablation plus the negative contribution of percentage ablated target area, is associated to each voxel, thus generating an ablation field map (Equation 1). The weights associated to damage $W_{d}$, target area ablation $W_{a}$ and additional tissue ablation $W_{e}$ can be tuned by clinicians to capture the desired trade-off between target ablation and collateral damage.

$$
S_{v}=-W_{a} * \frac{N_{a}}{N_{T}}+W_{d} * \frac{N_{d}}{N_{T}}+W_{e} * \frac{N_{r}}{N_{T}}
$$

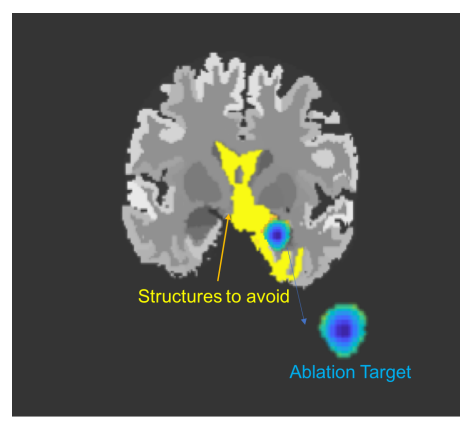

Fig. 1. A coronal slice of the generated ablation field is shown as an overlay of the brain parcellation. The ablation target region features a darker area corresponding to lower " $S_{v}$ " values, thus better ablation performances and less collateral damage.

Given the generated ablation field map, the ablation path can be optimized accordingly. Specifically, the search map section delimited by the tectal plate (which generally identifies the transition between the hippocampal body and tail) and the anterior border of the amygdala, is selected. Then, for each cross section, the first ranked voxel position in the corresponding ablation field map is included as part of the optimized ablation path, which is then smoothed using a convex optimization based technique [29] that accounts for the given curvature constraint. At this stage, we extend the

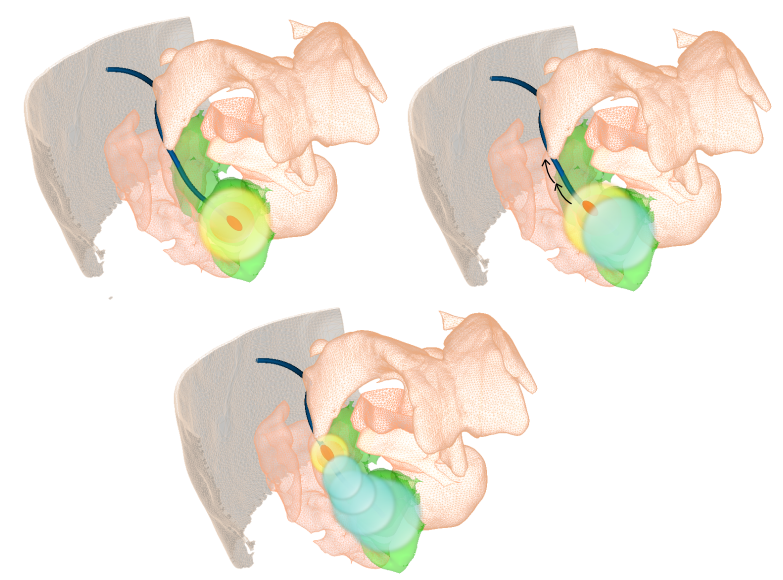

Fig. 2. The series shows LiTT performed along a curved path. The ablation field around the laser tip is represented by a yellow sphere. Starting from the deepest target point, the laser fiber is progressively extracted at a predefined step and the laser power is tuned to cover the desired laser ablation diameter. The overlapping ablation spheres finally result in a beam-shaped ablated volume (light blue)

optimization to the more complex case of a non-uniform $D_{a}$. This provides an improved LiTT clinical scenario in which clinicians could benefit from fine tuning the ablation diameter value at each step of the catheter insertion, within a range between 5 and $15 \mathrm{~mm}$, thus increasing their ability to precisely conform the ablation to the target structures. Given a predefined ablation pullback ( $7 \mathrm{~mm}$ is our default value), a second GPU accelerated algorithm is implemented to optimize the laser ablation diameter value for each step along the ablation path. In this process, each ablation step is represented as a series of overlapping spheres, thus approximating a cylindrical ablation volume around the given path (Figure 2). The spheres' radius is progressively increased in steps of 0.5 $\mathrm{mm}$ from 2.5 to $7.5 \mathrm{~mm}$. A score $S$ is associated to each cylinder, as in Equation 1, so that the first ranked cylinder dictates the value of the optimized $D_{a}$ associated to that portion of the ablation path. At the end of the computation, the optimized $D_{a}$ for each ablation step is provided. Additionally, the volume overlap with the amygdala, hippocampus, EnC, $\mathrm{PiCx}, \mathrm{PHG}, \mathrm{FuG}$ and $\mathrm{WM}$, as determined from the brain parcellation, is automatically calculated. Finally, the estimated ablation volumes are normalized by the preoperative volume to provide the percentage of ablation for each structure and an overall target $\%$ ablation as the sum of the contributions of all target structures.

2) AFT-connection: An adaptive fractal tree (AFT) based planner is used to find a path connecting the patient's skull to the initial pose of the ablation path. Surgical constraints for this portion of the path capture the angle of insertion with respect to the the skull normal, which should be close to perpendicular in order to prevent skidding of the cranial perforator, and the predefined safety margin from sensitive brain structures along the entire trajectory. In addition, the kinematic constraints of the flexible catheter require the generation of a curvaturebounded path which is assessed in terms of smoothness and length through a cost function. The AFT planner is a GPU- 
accelerated algorithm based on the propagation of a selfsimilar structure throughout the search space, resulting in a tree shape fractal. The basic fractal structure features a straight line and several arcs of circumference satisfying a given maximum curvature constraint, where the density and length can be tuned by the user. Obstacle-free branches that continuously connect a given target region back to the root of the tree constitute a feasible path for a nonholonomic steerable needle [34]. In this study, we are interested in trajectories matching not only the desired target position (corresponding to the first point of the ablation path) but also its orientation, in order to guarantee tangent continuity on the overall trajectory. To do so, we implemented some of the techniques discussed in [35], which optimize the choice of AFT parameters specifically for neurosurgery and provide different methods to take into account the goal pose accuracy, which includes both target position and target approach angle accuracy. A fractal tree is grown from an entry pose perpendicular to the patient's skull, then a "reachability volume" with the shape of a horn torus is placed in correspondence of the goal pose and directed towards the patient skull to filter only those samples laying within. The horn torus radius corresponds to the maximum needle achievable curvature and ensures that the AFT obstacle-free paths, laying within, meet the desired target pose and curvature constraint.

The full path obtained from the combination of the ablation path and the AFT-connection is finally smoothed to guarantee continuity and avoid potential misalignment. The generated paths are evaluated by mean of a normalized score $S_{p}$ (Equation 2), which corresponds to the weighted contribution of two terms: $S m$ and Risk for all $N$ path samples. The first corresponds to a cost function, introduced in [35], that measures the path smoothness in terms of overall curvature $\left(C_{i}\right)$, gradient of curvature between consecutive points $\left(C_{i+1}\right.$ - $C_{i}$ ) and length of the given trajectory (length). More linear and shorter paths are favoured to reduce the extent of tissue damage and facilitate the needle insertion. The second term is taken from the study by Li et al. [19] and captures the risk of a given path in terms of overall distance from vasculature and sulci, with a minimum distance Dist of $3 \mathrm{~mm}$. Paths that are further away from vessels are favoured, since they reduce the chance of a potential hemorrhage. Both terms are normalised within the $[0,1]$ range and their weight $W_{s}$ and $W_{r}$ can be tuned according to surgeon preference.

$$
\begin{aligned}
& S_{p}=W_{s} * S m+W_{r} * \text { Risk } \\
& S m=\sum_{i}^{N-1} \frac{\left|C_{i+1}-C_{i}\right|}{2 * \max _{\text {curv }}}+\sum_{i}^{n_{\text {seg }}} \frac{\left|C_{i}\right|}{\max _{\text {curv }}}+\frac{\text { length }}{\text { max }_{\text {length }}} \\
& \text { Risk }= \begin{cases}\sum_{i}^{N} \frac{10-\operatorname{Dist}(i)}{N(10-3)}, & \text { if } \operatorname{Dist}(i)>3 \\
1-\sum_{i}^{N} \frac{3-\operatorname{Dist}(i)}{3 * N}, & \text { if } \operatorname{Dist}(i) \leq 3\end{cases}
\end{aligned}
$$

\section{B. Dataset}

Following ethics approval provided by the National Research Ethics Service Committee, with approval reference 12/LO/0377, five patients from the National Hospital for Neurology and Neurosurgery epilepsy surgery program were included in this study. For each patient the following diagnostic images were provided: single T1 MPRAGE acquisition with a voxel size of $1 \mathrm{~mm}$ isotropic (TE/TR/TI = 3.1/7.4/400 ms; flip angle $11^{\circ}$; parallel imaging acceleration factor 2 ), wholebrain parcellation and synthetic CT (pseudo-CT), images were generated using geodesic information flow [36] and a multiatlas information propagation scheme [37], respectively.

\section{Simulations}

Patient-specific 3D models of the ventricular system, sulci, brainstem, PHG, FuG, AHC, EnC, WM and PicX are extracted from the whole brain parcellation. In particular, the brain stem (including LGN) was expanded by $7.5 \mathrm{~mm}$ to avoid any ablation related damage while ventricles, PHG, FuG, WM and sulci are merged with the vasculature segmentation to create an obstacle map, which is then expanded with a tunable safety margin, set as $1.5 \mathrm{~mm}$ by default (Figure 4). This takes into account both the needle occupancy and real-world uncertainties and it is more or less conservative depending on the geometry of the flexible tool considered. Currently used straight needles are about $0.8 \mathrm{~mm}$ in radius (i.e. Medtronic Visualase ${ }^{\mathrm{TM}}$ ), while flexible catheters are generally larger, reaching up to $2.5 \mathrm{~mm}$ in diameter [38], [28]. Similarly, 3D models of the amygdala, hippocampus, EnC and temporal PiCx constitute the ablation target area for the proposed curved CAP LiTT (Figure 3). The ablation path is calculated for each patient's anatomy, $W_{a}$ and $W_{c}$ are set to 1 and 0.5 respectively, while $W_{d}$ is progressively increased in steps of 0.5 starting from zero until the PHG damage rate reaches a maximum of $16 \%$. This threshold has been arbitrarily fixed looking at clinical accepted values and optimized straight LiTT CAP results from the literature, which show average PHG damage rate of $21 \%$ for manual insertions and $12 \%$ for straight CAP trajectories [20]. The ablation step is set to $7 \mathrm{~mm}$ by default and the best ranked $D_{a}$ is generated for each insertion step, as previously described. The overall $\%$ of damage and \% ablated target area, corresponding to the calculated ablation path, can be interactively assessed by neurosurgeons, and both a $3 \mathrm{D}$ rendering and a $2 \mathrm{D}$ overlay aid them during this process. A pseudo-CT is used to segment the patient's skull through simple threshold-based segmentation. A uniform mesh is then created from its surface, where the vertex coordinates represent possible entry points, and the associated normals represent the corresponding start approach vectors. Between these, a feasibility check with respect to the desired target pose is performed, as in [35]. This ascertains whether the start and target combination is feasible either in terms of maximum needle length or the maximum curvature constraint. The AFT-based planner runs over each entry pose to look for a connection with the given target pose, which coincides with the first pose of the ablation path. The resulting curvature-bounded and obstacle-free paths are merged with the previously calculated ablation path and ranked by means of the $S_{p}$ score (Equation 2). Finally, the first ranked path and the corresponding ablation and damage areas are offered to clinicians for review (Figure 5 and 6).

\section{RESULTS}

Following the implementation of a $5 \mathrm{~mm}$ to $15 \mathrm{~mm}$ variable laser ablation diameter, ablation results for the generated 


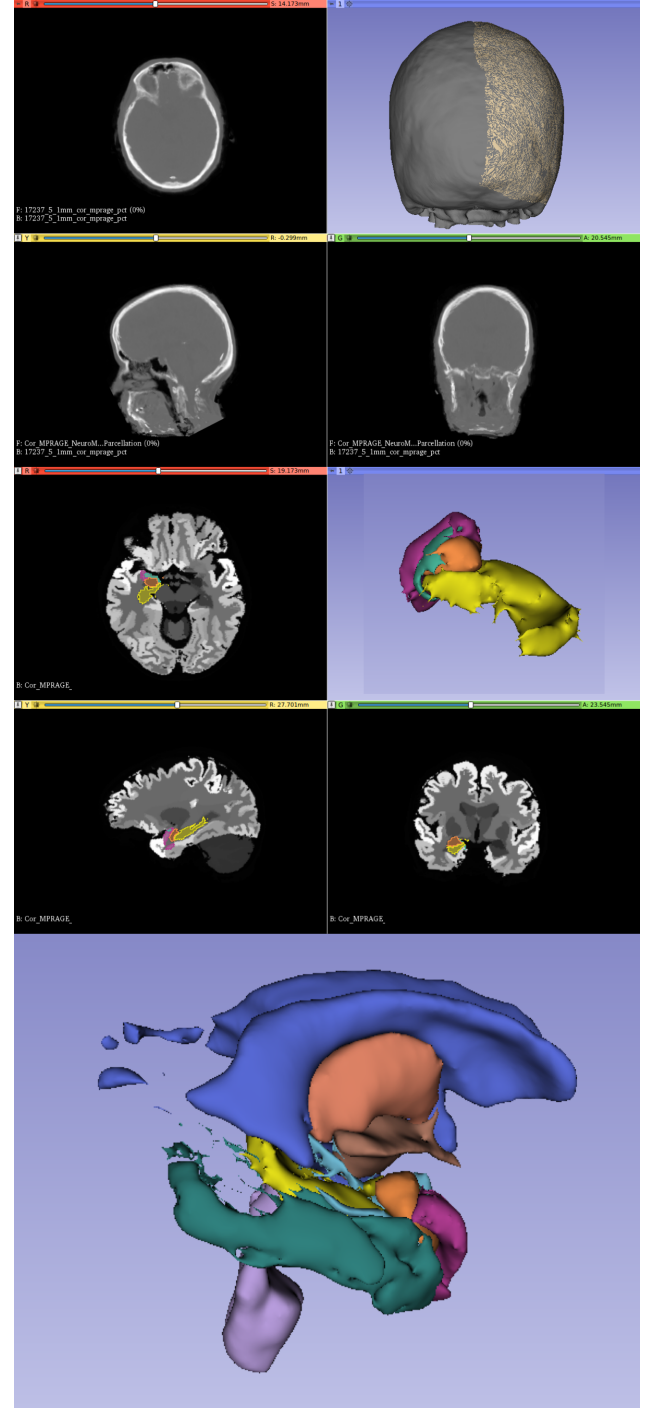

Fig. 3. (Top) The pseudo-CT is segmented through thresholding and the skull surface mesh of the selected hemisphere is considered. (Middle) The ablation target regions are extracted from the patient's MRI parcellation: hippocampus (yellow), amygdala (orange), temporal piriform cortex (light green) and entorhinal cortex (purple). (Bottom) Sensitive brain structures surrounding the ablation area are also extracted from the parcellation: ventricles (blue), brainstem (violet), thalamus (salmon) and the PHG (light blue) on the back with respect to the FuG (dark green).

curved CAP trajectories are compared to their straight counterparts, generated with the machine learning based straight CAP described in [20]. A one sample t-test is used to analyse the difference between the means while an F-test evaluates the difference in the standard deviations. As illustrated in Figure 7 and Table I: curved CAP results in a significant improvement in terms of greater $\%$ ablated, on amygdala, hippocampus, EnC (t-test, $\mathrm{p} \leq 0.05$ ) and on the overall target area (t-test, $\mathrm{p} \leq 0.01)$; While a higher standard deviation was registered for the amygdala (F-test, $\mathrm{p} \leq 0.05$ ) with respect to straight insertions [20]. Additionally, $\mathrm{PiCx}$ ablation is included for the first time during LiTT planning optimization, resulting in a great rate of ablation of its temporal portion for both curved and straight CAP, with no statistical difference.

On the other hand, damage to sensitive structures (Table

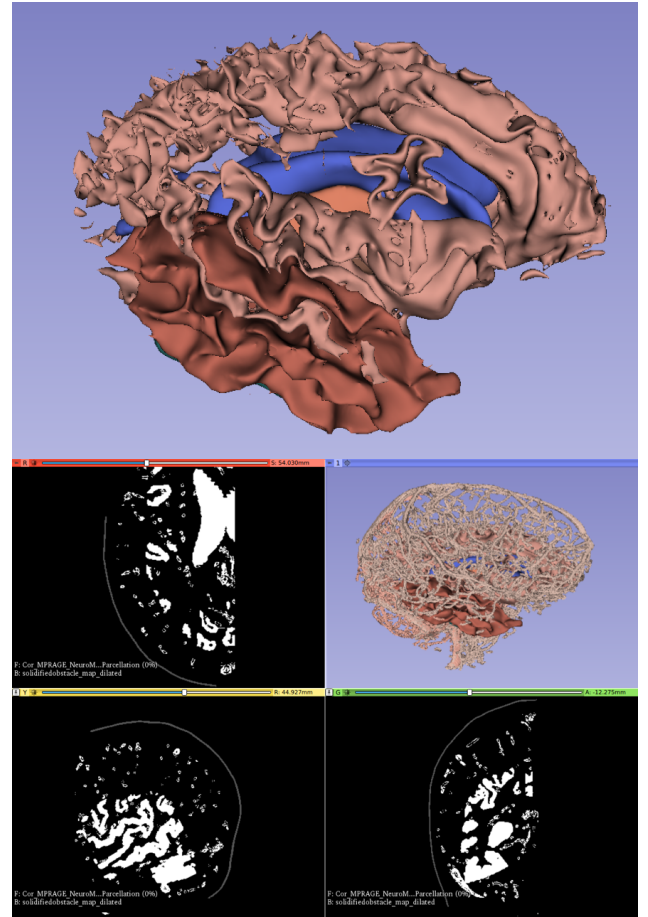

Fig. 4. Sulci, temporal white matter and vasculature are part of the obstacle map. In the bottom, the resulting binary obstacle map is visualised in the three standard 2D views. The obstacles (white regions) have already been dilated by a safety margin $(1.5 \mathrm{~mm}$ in this example).

I: \% ablation of non target areas) is minimised. The PHG ablation maximum tolerance was set to $16 \%$, as this value has been shown to be acceptable both in past manual and automated LiTT planning results [20], [19], with a significant reduction in $\%$ of ablation variance in the case of curved CAP (F-test, $\mathrm{p} \leq 0.05$ ). WM and FuG damage rates are small for both curved and straight method, while the FuG standard deviation is statistically lower for curved CAP (F-test, $\mathrm{p} \leq$ $0.01)$. The smaller standard deviation, associated to $\%$ ablation of non target areas, suggests that greater flexibility could lead to higher CAP robustness.

With respect to safety metrics (Table I: safety metrics), curved CAP-planned trajectories feature not significantly higher average intracerebral length (Length) with respect to straight paths. On the other hand, curved CAP trajectories resulted in a highly significant reduction in the computed overall risk (Risk) score (t-test, $\mathrm{p} \leq 0.001)$, based on a cumulative distance from critical structures (sulci and vasculature) along the entire length of the trajectory, thus reducing the potential risk of haemorrhage for patients. Finally, the cost function average value $S_{m}$ for curved CAP is equal to 0.6 , with a variance of \pm 0.03 .

\section{DISCUSSION}

The proposed curved LiTT algorithm presents enhanced flexibility, thus greater ability to cope with several constraints associated to better patient outcomes. The generated trajectories begin perpendicular to the patient's skull and advance through the brain meeting the hard constraints on the desired safety distance from all the predefined structures. A greater $\%$ 

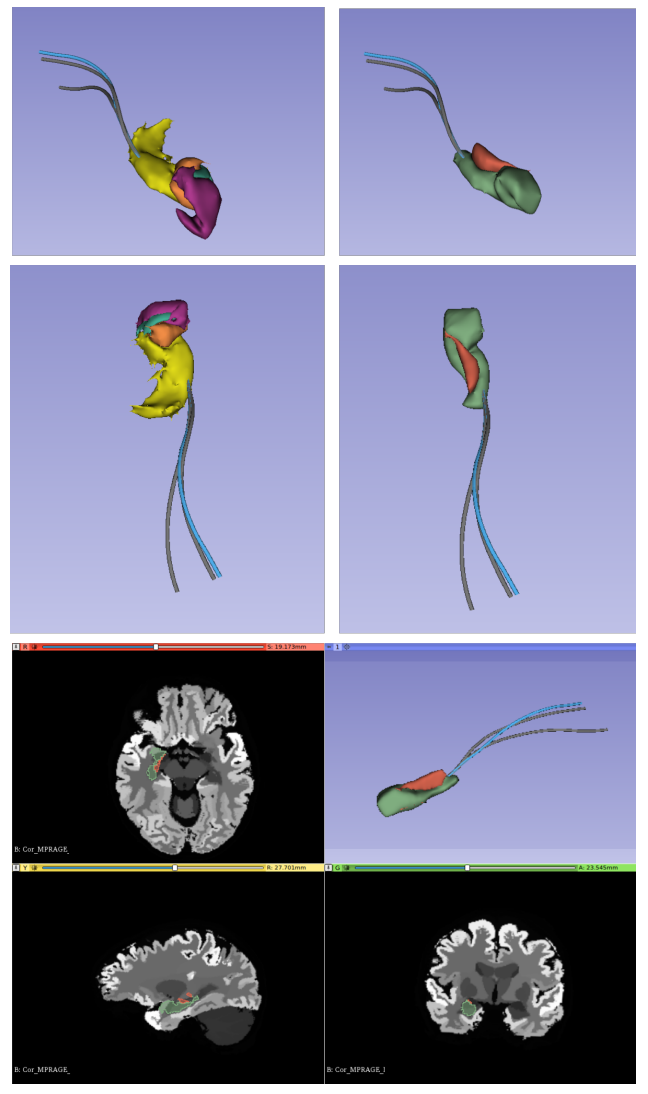

Fig. 5. Three of the generated curved and optimised paths are shown in the top left column, together with the segmented ablation target regions; while the ablated target area (top right, green volume) and the collateral damage (top right, red volume) show the actual performances associated to the best ranked path (blue).

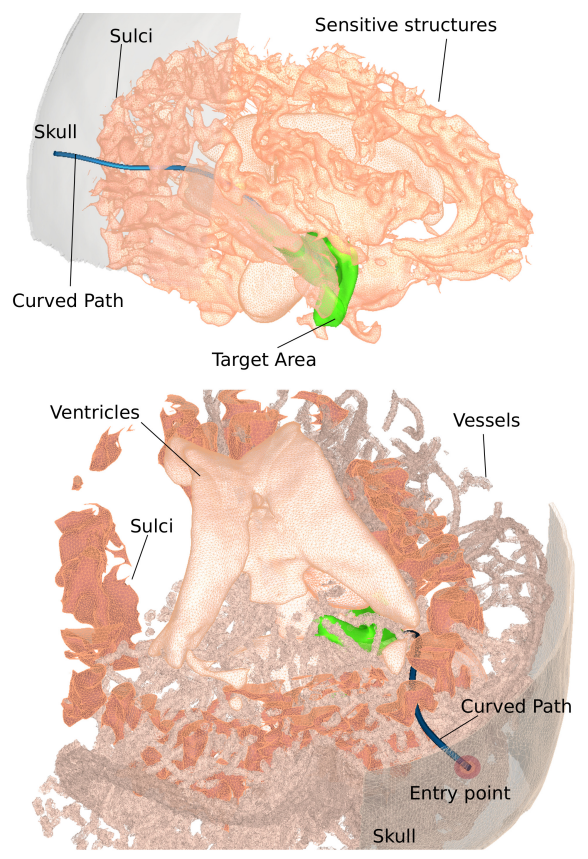

Fig. 6. The best ranked path is shown to be collision-free within the intricate brain obstacle map. The map is cropped axially (bottom image) at the level of the specified path to show its sensitive structures avoidance.

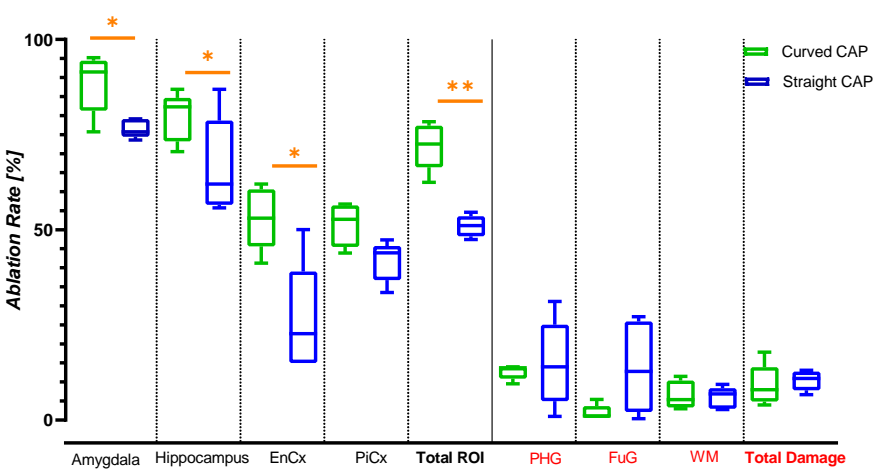

Fig. 7. Box-plots of curved CAP \% ablation (green) versus straight CAP results (blue). The statistical significance resulting from one sample t-test is shown using the $\mathrm{p}$-value standard convention: * for $\mathrm{p} \leq 0.05$, ** for $\mathrm{p} \leq 0.01$, $* * * \mathrm{p} \leq 0.001, * * * * \mathrm{p} \leq 0.0001$.

TABLE I

\% Ablation Target Areas

\begin{tabular}{|c|c|c|c|c|}
\hline \multicolumn{2}{|c|}{ Curved CAP } & Straight $C A P$ & t-test & $F$-test \\
\hline Hipросатриs & $79.6 \pm 6.4 \%$ & $66.5 \pm 12.8 \%$ & $*$ & $\mathrm{~ns}$ \\
\hline Amygdala & $88.6 \pm 7.8 \%$ & $76.6 \pm 2.4 \%$ & * & $*$ \\
\hline$E n C$ & $53.1 \pm 8.2 \%$ & $26.1 \pm 14.5 \%$ & $*$ & $\mathrm{~ns}$ \\
\hline$P i C x$ & $51.3 \pm 5.6 \%$ & $41.8 \pm 5.3 \%$ & $\mathrm{~ns}$ & $\mathrm{~ns}$ \\
\hline Total & $72 \pm 6.2 \%$ & $50.9 \pm 2.8 \%$ & ** & $\mathrm{ns}$ \\
\hline
\end{tabular}

$\%$ Ablation of Non Target Areas (collateral damage)

\begin{tabular}{|c|c|c|c|c|}
\hline & urved $C A P$ & Straight CAP & t-test & $F$-tes \\
\hline$P H G$ & $12.7 \pm 1.9 \%$ & $14.8 \pm 11.3 \%$ & $\mathrm{~ns}$ & \\
\hline$W M$ & $6.6 \pm 3.7 \%$ & $5.9 \pm 2.8$ & $\mathrm{~ns}$ & ns \\
\hline$F u G$ & $1.9 \pm 2 \%$ & $13.8 \pm 11.9$ & $\mathrm{~ns}$ & $* *$ \\
\hline Total & $9.1 \pm 5.3 \%$ & $10.4 \pm 2.6$ & $\mathrm{~ns}$ & $\mathrm{~ns}$ \\
\hline
\end{tabular}

Safety Metrics

\begin{tabular}{l|llcc} 
& Curved CAP & Straight CAP & t-test & F-test \\
\hline Length & $111 \pm 7.5 \mathrm{~mm}$ & $100 \pm 9.1 \mathrm{~mm}$ & $\mathrm{~ns}$ & $\mathrm{~ns}$ \\
\hline Risk & $0.4 \pm 0.1$ & $1.3 \pm 0.1$ & $* * * *$ & $\mathrm{~ns}$ \\
\hline$S_{m}$ & $0.6 \pm 0.03$ & NA & NA & NA \\
\hline
\end{tabular}

Curved CAP versus straight CAP Performance. Statistically significant results from one sample t-test and F-test are shown using the p-value standard convention: $*$ for $\mathrm{p} \leq 0.05, * *$ for $\mathrm{p} \leq 0.01, * * *$ for $\mathrm{p} \leq 0.001, * * * *$ for $\mathrm{p} \leq 0.0001$, ns for not significant and NA for not comparable available data.

ablation target area at reduced damage of PHG and nearby maesial sensitive structures is shown. The small standard deviation associated with both target area ablation and damage to sensitive structures demonstrates that curved ablation paths can handle different anatomies with equal performance. Also, the greater $\mathrm{EnCx}$, amygdala and hippocampus ablation coverage and the inclusion of the $\mathrm{PiCx}$ at no expense of further PHG damage or smaller hippocampus ablation confirms the potential of steerable needles for LiTT. Furthermore, the ability of moving through a cluttered environment avoiding obstacles corresponds to smaller registered risk metrics and thus a reduced risk for patients. It is also worth considering that steerable needles are robotically driven systems that can be guided in real-time through soft-tissue to allow for small adjustments with respect to the preoperative plan, if needed. Their intra-operative flexibility could compensate for possible misalignments and tissue deformations, due to real-world uncertainties, which are responsible for erroneous placement 
and the need for follow-on LiTT surgery in up to $46 \%$ of patients [39]. Finally, performing the desired ablation with a single curved insertion may reduce the overall error rate which, especially in the case of multiple straight passes, equals the sum of all contributions. However, the generally larger diameter associated to current flexible catheters (e.g.[40], [41]) could potentially cause more damage along the track, which means that further miniaturisation of this technology would be desirable. The EDEN2020 setup has already been tested within a standard surgical workflow during preclinical animal trials, demonstrating successful real-time performance and high target accuracy [28]. Following the results of this study, a new version of the existing EDEN2020 PBN, with smaller footprint and higher steerability, will be developed. The proposed curved LiTT CAP algorithm will be included as an additional preoperative planner module within the EDEN2020 frontend interface. Autonomous path following under surgeon's supervision can be performed through adaptive path-following control [42]. To track the needle inside tissue, a system based on Fiber Bragg Gratings (FBGs) was being developed to track the needle tip [43], as well as to sense the shape of each of the four needle segments, while intraoperative Ultrasound is used to provide information about real-time tissue deformation [44]. The application of steerable needles in ablative therapies could also be beneficial in situations that require multi-trajectory planning, such as corpus callosotomy for generalized epilepsy. [45].

\section{CONCLUSION}

This is the first fully automated CAP method for the generation of curved LiTT trajectories that maximizes both the ablation of mesial temporal target areas (hippocampus, amygdala, $\mathrm{EnC}$ and $\mathrm{PiCx}$ ) and patient safety metrics, while minimizing collateral damage to nearby structures (PHG, WM and $\mathrm{FuG})$. We have also presented the optimization of a variable ablation diameter field ranging between 5 and 15 $\mathrm{mm}$ diameter along the generated curved ablation trajectory and demonstrated that LiTT procedures could benefit from steerable needle technologies by tailoring the insertion path to the subject-specific anatomy. CAP methods provide an objective means of planning LiTT procedures that may overcome the heterogeneity existing between different surgeons and institutions. Future studies using larger datasets will be required to further assess and validate the exciting potential of the proposed method.

\section{REFERENCES}

[1] E. P. Westebring - van der Putten, R. H. M. Goossens, J. J. Jakimowicz, and J. Dankelman, "Haptics in minimally invasive surgery - a review," Minimally Invasive Therapy \& Allied Technologies, vol. 17, no. 1, pp. 3-16, jan 2008.

[2] M. A. Audette, S. P. A. Bordas, and J. E. Blatt, "Robotically Steered Needles: A Survey of Neurosurgical Applications and Technical Innovations," Robotic Surgery: Research and Reviews, vol. 7, p. 1, 2020.

[3] H. J. Marcus, V. N. Vakharia, S. Ourselin, J. Duncan, M. Tisdall, and K. Aquilina, "Robot-assisted stereotactic brain biopsy: Systematic review and bibliometric analysis," Child's Nervous System, vol. 34, no. 7, pp. 1299-1309, jul 2018.

[4] A. Hong, Q. Boehler, R. Moser, A. Zemmar, L. Stieglitz, and B. J. Nelson, "3D path planning for flexible needle steering in neurosurgery," Tech. Rep. 4, 2019.
[5] A. J. Petruska, F. Ruetz, A. Hong, L. Regli, O. Surucu, A. Zemmar, and B. J. Nelson, "Magnetic needle guidance for neurosurgery: Initial design and proof of concept," in 2016 IEEE International Conference on Robotics and Automation (ICRA). IEEE, may 2016, pp. 4392-4397.

[6] A. Segato, V. Pieri, A. Favaro, M. Riva, A. Falini, E. De Momi, and A. Castellano, "Automated Steerable Path Planning for Deep Brain Stimulation Safeguarding Fiber Tracts and Deep Gray Matter Nuclei," Frontiers in Robotics and AI, vol. 6, p. 70, aug 2019.

[7] J. Burgner, P. J. Swaney, R. A. Lathrop, K. D. Weaver, and R. J. Webster, "Debulking From Within: A Robotic Steerable Cannula for Intracerebral Hemorrhage Evacuation," IEEE Transactions on Biomedical Engineering, vol. 60, no. 9, pp. 2567-2575, sep 2013.

[8] B. Konh, N. V. Datla, and P. Hutapea, "Dynamic estimation of an active surgical needle deflection for brachytherapy procedures," in 2014 40th Annual Northeast Bioengineering Conference (NEBEC). IEEE, apr 2014, pp. 1-2.

[9] W. Zhan, D. Dini, and F. R. y. Baena, "Transport of Chemotherapeutic Agents in Anisotropic Tissue under Convection Enhanced Delivery for Localised Treatment," 2019.

[10] R. L. Hood, R. T. Andriani, T. E. Ecker, J. L. Robertson, and C. G. Rylander, "Characterizing Thermal Augmentation of Convection-Enhanced Drug Delivery with the Fiberoptic Microneedle Device," Engineering, vol. 1 , no. 3, pp. 344-350, sep 2015.

[11] C. Hoppe, J.-A. Witt, C. Helmstaedter, T. Gasser, H. Vatter, and C. E. Elger, "Laser interstitial thermotherapy (LiTT) in epilepsy surgery." Seizure, vol. 48, pp. 45-52, may 2017.

[12] F. Cendes, "Mesial temporal lobe epilepsy syndrome: an updated overview," Journal of Epilepsy and Clinical Neurophysiology, vol. 11, no. 3, pp. 141-144, 2005.

[13] C. Wu, W. J. Jermakowicz, S. Chakravorti, I. Cajigas, A. D. Sharan, J. R. Jagid, C. M. Matias, M. R. Sperling, R. Buckley, A. Ko, J. G. Ojemann, J. W. Miller, B. Youngerman, S. A. Sheth, G. M. McKhann, A. W. Laxton, D. E. Couture, G. S. Popli, A. Smith, A. D. Mehta, A. L. Ho, C. H. Halpern, D. J. Englot, J. S. Neimat, P. E. Konrad, E. Neal, F. L. Vale, K. L. Holloway, E. L. Air, J. Schwalb, B. M. Dawant, and P. D'Haese, "Effects of surgical targeting in laser interstitial thermal therapy for mesial temporal lobe epilepsy: A multicenter study of 234 patients," Epilepsia, vol. 60, no. 6, p. epi.15565, may 2019.

[14] D. L. Drane, D. W. Loring, N. L. Voets, M. Price, J. G. Ojemann, J. T. Willie, A. M. Saindane, V. Phatak, M. Ivanisevic, S. Millis, S. L. Helmers, J. W. Miller, K. J. Meador, and R. E. Gross, "Better object recognition and naming outcome with MRI-guided stereotactic laser amygdalohippocampotomy for temporal lobe epilepsy." Epilepsia, vol. 56, no. 1, pp. 101-13, jan 2015.

[15] M. Galovic, I. Baudracco, E. Wright-Goff, G. Pillajo, P. Nachev, B. Wandschneider, F. Woermann, P. Thompson, S. Baxendale, A. W. McEvoy, M. Nowell, M. Mancini, S. B. Vos, G. P. Winston, R. Sparks, F. Prados, A. Miserocchi, J. de Tisi, L. A. Van Graan, R. Rodionov, C. Wu, M. Alizadeh, L. Kozlowski, A. D. Sharan, L. G. Kini, K. A. Davis, B. Litt, S. Ourselin, S. L. Moshé, J. W. A. Sander, W. Löscher, J. S. Duncan, and M. J. Koepp, "Association of Piriform Cortex Resection With Surgical Outcomes in Patients With Temporal Lobe Epilepsy.” JAMA neurology, vol. 76, no. 6, pp. 690-700, 2019.

[16] W. J. Jermakowicz, A. M. Kanner, S. Sur, C. Bermudez, P.-F. D'Haese, J. P. G. Kolcun, I. Cajigas, R. Li, C. Millan, R. Ribot, E. A. Serrano, N. Velez, M. R. Lowe, G. J. Rey, and J. R. Jagid, "Laser thermal ablation for mesiotemporal epilepsy: Analysis of ablation volumes and trajectories." Epilepsia, vol. 58, no. 5, pp. 801-810, 2017.

[17] H. Waseem, K. E. Osborn, M. R. Schoenberg, V. Kelley, A. Bozorg, D. Cabello, S. R. Benbadis, and F. L. Vale, "Laser ablation therapy: An alternative treatment for medically resistant mesial temporal lobe epilepsy after age 50." Epilepsy \& behavior : E\&B, vol. 51, pp. 152-7, oct 2015.

[18] V. N. Vakharia, R. Sparks, K. Li, A. G. O'Keeffe, A. Miserocchi, A. W. McEvoy, M. R. Sperling, A. Sharan, S. Ourselin, J. S. Duncan, and C. Wu, "Automated trajectory planning for laser interstitial thermal therapy in mesial temporal lobe epilepsy," Epilepsia, vol. 59, no. 4, pp. 814-824, 2018.

[19] K. Li, V. N. Vakharia, R. Sparks, L. G. França, A. Granados, A. W. McEvoy, A. Miserocchi, M. Wang, S. Ourselin, and J. S. Duncan, "Optimizing Trajectories for Cranial Laser Interstitial Thermal Therapy Using Computer-Assisted Planning: A Machine Learning Approach," Neurotherapeutics, vol. 16, no. 1, pp. 182-191, 2019.

[20] V. N. Vakharia, R. E. Sparks, K. Li, A. G. O'Keeffe, F. Pérez-García, L. G. França, A. L. Ko, C. Wu, J. P. Aronson, B. E. Youngerman, A. Sharan, G. McKhann, S. Ourselin, and J. S. Duncan, "Multicenter 
validation of automated trajectories for selective laser amygdalohippocampectomy," Epilepsia, vol. 60, no. 9, pp. 1949-1959, 2019.

[21] D. B. Comber, E. B. Pitt, H. B. Gilbert, M. W. Powelson, E. Matijevich, J. S. Neimat, R. J. Webster, and E. J. Barth, "Optimization of Curvilinear Needle Trajectories for Transforamenal Hippocampotomy," Operative Neurosurgery, vol. 13, no. 1, pp. 15-22, feb 2017.

[22] S. S. Grewal, M. A. Alvi, V. M. Lu, W. Wahood, G. A. Worrell, W. Tatum, R. E. Wharen, and J. J. Van Gompel, "Magnetic ResonanceGuided Laser Interstitial Thermal Therapy Versus Stereotactic Radiosurgery for Medically Intractable Temporal Lobe Epilepsy: A Systematic Review and Meta-Analysis of Seizure Outcomes and Complications." World neurosurgery, vol. 122, pp. e32-e47, feb 2019.

[23] C. Wu, W. J. Jermakowicz, S. Chakravorti, I. Cajigas, A. D. Sharan, J. R. Jagid, C. M. Matias, M. R. Sperling, R. Buckley, A. Ko, J. G. Ojemann, J. W. Miller, B. Youngerman, S. A. Sheth, G. M. McKhann, A. W. Laxton, D. E. Couture, G. S. Popli, A. Smith, A. D. Mehta, A. L. Ho, C. H. Halpern, D. J. Englot, J. S. Neimat, P. E. Konrad, E. Neal, F. L. Vale, K. L. Holloway, E. L. Air, J. Schwalb, B. M. Dawant, and P.-F. D'Haese, "Effects of surgical targeting in laser interstitial thermal therapy for mesial temporal lobe epilepsy: A multicenter study of 234 patients." Epilepsia, vol. 60, no. 6, pp. 1171-1183, 2019.

[24] Y. Chen, M. E. Poorman, D. B. Comber, E. B. Pitt, C. Liu, I. S. Godage, H. Yu, W. A. Grissom, E. J. Barth, and R. J. Webster, "Treating Epilepsy via Thermal Ablation: Initial Experiments With an MRIGuided Concentric Tube Robot," in 2017 Design of Medical Devices Conference. American Society of Mechanical Engineers, apr 2017.

[25] I. Robert J. Webster, J. S. Kim, N. J. Cowan, G. S. Chirikjian, and A. M. Okamura, "Nonholonomic modeling of needle steering," The International Journal of Robotics Research, vol. 25, no. 5-6, pp. 509525, 2006.

[26] L. Frasson, S. Y. Ko, A. Turner, T. Parittotokkaporn, J. F. Vincent, and F. Rodriguez y Baena, "STING: a soft-tissue intervention and neurosurgical guide to access deep brain lesions through curved trajectories." Proceedings of the Institution of Mechanical Engineers. Part H, Journal of engineering in medicine, vol. 224, no. 6, pp. 775-88, jun 2010.

[27] D. S. Minhas, J. A. Engh, M. M. Fenske, and C. N. Riviere, "Modeling of needle steering via duty-cycled spinning," Annual International Conference of the IEEE Engineering in Medicine and Biology - Proceedings, pp. 2756-2759, 2007

[28] R. Secoli, E. Matheson, S. Galvan, M. Pinzi, T. Watts, A. Donder, and F. Rodriguez y Baena, "A Modular Robotic Platform for Precision Neurosurgery with a Programmable Bevel-Tip Needle," in The International Journal of Robotics Research, 2020.

[29] R. S. S. G. F. R. y. B. M. Pinzi, T. Watts, "Adaptive Path Replanning for Orientation-constrained Needle Steering." Transaction of Biomedical Engineering, 2020.

[30] P. Li, S. Jiang, D. Liang, Z. Yang, Y. Yu, and W. Wang, "Modeling of path planning and needle steering with path tracking in anatomical soft tissues for minimally invasive surgery," Medical Engineering \& Physics, vol. 41, pp. 35-45, mar 2017.

[31] D. B. Comber, E. B. Pitt, H. B. Gilbert, M. W. Powelson, E. Matijevich, J. S. Neimat, R. J. Webster, and E. J. Barth, "Optimization of Curvilinear Needle Trajectories for Transforamenal Hippocampotomy," Operative Neurosurgery, vol. 13, no. 1, p. 1, jul 2016.

[32] T. Watts, R. Secoli, and F. R. y. Baena, "A Mechanics-Based Model for 3-D Steering of Programmable Bevel-Tip Needles," IEEE Transactions on Robotics, vol. 35, no. 2, pp. 371-386, apr 2019.

[33] M. Terzano, D. Dini, F. Rodriguez y Baena, A. Spagnoli, and M. Oldfield, "An adaptive finite element model for steerable needles," Biomechanics and Modeling in Mechanobiology, vol. 19, no. 5, pp. 1809-1825, oct 2020.

[34] F. Liu, A. Garriga-Casanovas, R. Secoli, and F. Rodriguez y Baena, "Fast and Adaptive Fractal Tree-Based Path Planning for Programmable Bevel Tip Steerable Needles," IEEE Robotics and Automation Letters, vol. 1, no. 2, pp. 601-608, jul 2016.

[35] M. Pinzi, S. Galvan, and F. Rodriguez y Baena, "The Adaptive Hermite Fractal Tree (AHFT): a novel surgical 3D path planning approach with curvature and heading constraints," International Journal of Computer Assisted Radiology and Surgery, vol. 14, no. 4, pp. 659-670, apr 2019

[36] M. J. Cardoso, M. Modat, R. Wolz, A. Melbourne, D. Cash, D. Rueckert, and S. Ourselin, "Geodesic Information Flows: Spatially-Variant Graphs and Their Application to Segmentation and Fusion." IEEE transactions on medical imaging, vol. 34, no. 9, pp. 1976-88, sep 2015.

[37] N. Burgos, M. J. Cardoso, M. Modat, S. Pedemonte, J. Dickson, A. Barnes, J. S. Duncan, D. Atkinson, S. R. Arridge, B. F. Hutton, and S. Ourselin, "Attenuation correction synthesis for hybrid PET-MR scanners." Medical image computing and computer-assisted intervention
: MICCAI ... International Conference on Medical Image Computing and Computer-Assisted Intervention, vol. 16, no. Pt 1, pp. 147-54, 2013.

[38] L. Frasson, S. Y. Ko, A. Turner, T. Parittotokkaporn, J. F. Vincent, and F. Rodriguez Y Baena, "STING: A soft-tissue intervention and neurosurgical guide to access deep brain lesions through curved trajectories," Proceedings of the Institution of Mechanical Engineers, Part H: Journal of Engineering in Medicine, vol. 224, no. 6, pp. 775-788, 2010.

[39] E. Widjaja, T. Papastavros, B. Sander, C. Snead, and P. Pechlivanoglou, "Early economic evaluation of MRI-guided laser interstitial thermal therapy (MRgLITT) and epilepsy surgery for mesial temporal lobe epilepsy," PLOS ONE, vol. 14, no. 11, 2019

[40] L. Frasson, F. Ferroni, S. Y. Ko, G. Dogangil, and F. Rodriguez y Baena, "Experimental evaluation of a novel steerable probe with a programmable bevel tip inspired by nature," Journal of Robotic Surgery, vol. 6, no. 3, pp. 189-197, sep 2012.

[41] T. Watts, R. Secoli, and F. R. y. Baena, "A Mechanics-Based Model for 3-D Steering of Programmable Bevel-Tip Needles," IEEE Transactions on Robotics, vol. 35, no. 2, pp. 371-386, apr 2019.

[42] R. Secoli, F. Rodriguez, and Baena, "Experimental validation of curvature tracking with a programmable bevel-tip steerable needle," in 2018 International Symposium on Medical Robotics (ISMR). IEEE, mar 2018, pp. 1-6.

[43] F. Khan, A. Donder, S. Galvan, F. R. Y. Baena, and S. Misra, "Pose Measurement of Flexible Medical Instruments Using Fiber Bragg Gratings in Multi-Core Fiber," IEEE Sensors Journal, vol. 20, no. 18, pp. 10955-10 962, sep 2020.

[44] R. Göbl, N. Navab, and C. Hennersperger, "SUPRA: open-source software-defined ultrasound processing for real-time applications," International Journal of Computer Assisted Radiology and Surgery, vol. 13, no. 6, pp. 759-767, 2018 .

[45] V. N. Vakharia, R. E. Sparks, S. B. Vos, Y. Bezchlibnyk, A. D. Mehta, J. T. Willie, C. Wu, A. Sharan, S. Ourselin, and J. S. Duncan, "Computerassisted planning for minimally invasive anterior two-thirds laser corpus callosotomy: A feasibility study with probabilistic tractography validation." NeuroImage. Clinical, p. 102174.

Marlene Pinzi received the M.Eng. degree in Biomedical Engineering from "Politecnico di Milano" in 2016 and she is currently pursuing the Ph.D. degree at the Mechatronics in Medicine laboratory, Imperial College London. Her research focus on an intelligent path planner for surgical steerable needles.

Brian Hwang received his M.D. from Columbia University College of Physicians and Surgeons and completed a functional neurosurgery fellowship at Johns Hopkins. He is currently serving as chief neurosurgery resident at Johns Hopkins Department of Neurosurgery. His research focus includes epilepsy and neurosurgical innovation, technology, and device development.

William Anderson received his Ph.D. in Physics from Princeton in 1997 and M.D.from Johns Hopkins School of Medicine in 2001. As a functional neurosurgeon at Johns Hopkins Hospital, he specializes in the treatment of epilepsy, movement disorders, and spasticity. His research interests include computational modeling of seizures, memory encoding, and development of novel advanced neuromodulation techniques.

Ferdinando Rodriguez y Baena received a $\mathrm{Ph}$.D. degree in medical robotics in 2004. He is now Professor of Medical Robotics in the Department of Mechanical Engineering at Imperial College, where he leads the Mechatronics in Medicine Laboratory. He is also the Engineering Director of the Hamlyn Centre. His current research interests include the application of mechatronic systems to medicine.

John Duncan since 1989 he has been a Consultant Neurologist specialising in epilepsy, at the National Hospital for Neurology and Neurosurgery, Queen Square, London and at Epilepsy Society in Chalfont St Peter, UK, and leads the epilepsy imaging - surgery programme. He was appointed Professor of Neurology at the UCL Institute of Neurology in 1998. In 2004 he received the annual Clinical Research recognition award of the American Epilepsy Society. He is past-President of the UK chapter of ILAE. In 2005 he was elected Ambassador for Epilepsy and to be a Fellow of the Academy of Medical Sciences.

Vejay Vakharia is a neurosurgical fellow at The National Hospital for Neurology and Neurosurgery. He has a Ph.D. in epilepsy surgery with an interest in neuroimaging and computer-assisted planning for SEEG, brain biopsy and LiTT applications. 